\title{
Endothelial progenitor cells in ageing
}

"The senescence status of somatic stem/progenitor cells contributes to ageing process", and "an individual is as old as old are its stem cells". These quotes represent the concepts, which are actually object of investigations of researchers involved in ageing studies. By discussing on the fundamental cause of ageing, they actually use these expressions. Cotemporally, this recent evidence reinforces the concept, that the biological age is different from the chronological age. It includes the real speed of ageing of cells, tissues and organs of an organism, and consequently it indicates the real age of each individual. For this reason its estimation is very crucial, but co-temporally very complex. The identification of parameters, which can contribute to estimate the biological age, is a cutting-edge issue in the field of ageing. In this context, studies focussed on the involvement of stem/progenitor cells in degenerative ageing process should be encouraged. Stem/progenitor cell activity gradually decreases with advancing age. In particular, it has been evidenced that adult stem/progenitor cells reside in a quiescent state for prolonged periods of time, during which they may accumulate damages induced by the wear and tear of continued metabolic activity. As result their senescence or apoptosis can occur, as well as the decline in their ability to replace damaged cells. This determines an alteration of both homeostasis and function of tissues and organs, and the evocation of their biological ageing, which, in a prospective vision, can cause the onset of age-related diseases. Accordingly, the principal role of stem/progenitor cells is to provide a supply of new cells to keep the body in good functional condition, delaying the rate of accumulation of dysfunctions and frailty. Frailty is, in turn, one of the major risk factor for the onset of age-related diseases, as well as for death.

As consequence, it is emerging the research on stem/progenitor cell senescence and quiescence as an innovative field in the study of age-related diseases, and the recent findings obtained appear to be very promising. Indeed, new insights on quiescent status of stem/progenitor cells have been achieved, even if the interest of researchers is until now in a baby status and limited only to some groups. In particular, they suggest that the state of quiescence of the major number of adult stem/progenitor cells is not a default status, determined by challenges to continued proliferation, such as nutrient deprivation or contact inhibition, as previously supposed, but rather than it is actually considered as a status adopted by stem/progenitor cells to preserve key functional features. This status appears, hence, to be reversible. Indeed, recent insights into the epigenetic, transcriptional and post-transcriptional control of quiescence suggest that quiescence is an actively maintained state, in which signaling pathways are involved in maintaining a poised state that allows rapid activation. Deciphering the molecular mechanisms regulating adult stem/progenitor cell quiescence may increase our understanding of tissue regeneration mechanisms and how they are dy-sregulated in pathological conditions and in ageing. This might represent a flywheel for the development of therapies for slowing the rate of ageing, by modulating stem/progenitor cell activity/quiescence/senescence. In this contest, of special interest are recent studies, which have revealed a great degree of plasticity intercellular and organism ageing. Investigations on genetic dissection of the ageing process have revealed, that the manipulation of specific signaling pathways (e.g., insulin/IGF-1, mTOR, AMPK, and sirtuins) and environmental interventions (e.g., caloric restriction) are able to modulate the ageing process in multicellular organisms. Other groups have explored a very innovative anti-ageing approach, which might generate a very revolution in the field of translation medicine, or more precisely for regenerative medicine. It is based on connecting the circulatory systems of two organisms of different ages (i.e., heterochronic parabiosis experiments). The actual investigations performed have elegantly demonstrated, that exposing old tissues and organs to a young circulatory environment can rejuvenate tissue-specific stem/progenitor cells, leading to a youthful state, which is characterized by functional and regenerative improvements. This form of ageing plasticity has been observed in complex tissues, including skin, liver, muscle and bone marrow, and involves the modulation of evolutionally conserved signaling pathways, including Notch, Wnt and TGF- $\beta$ signaling pathways by young circulatory signals. The promising data obtained are leading to hypothesize of performing clinical human trials. Accordingly, encouraging is the unique the clinical human trial initiated at Stanford University to test the effects of young plasma on patients with Alzheimer's disease (Clinical Trial Identifier NCT02256306). The identification of circulatory signals, which rejuvenate ageing phenotypes is currently becoming the focus of an increasing number of laboratories around the world and, despite this challenge, several candidate molecules have been proposed, such as growth differentiation factor 11 (GDF11). 
However, contradictory observations have been recently made by independent groups regarding the potential of GDF11 as antiageing molecule, indicating that further studies will be necessary to continue this quest.

Thus, future and more intensive studies are necessary. However, researchers in this field have a strong incentive to address all the issues associated with stem/progenitor cell senescence/quiescence, since their interest is to treat age-related diseases with innovative or first generation cell based therapies. As life expectancy in Western countries continues to increase, so does the risk of experiencing comorbidity and disability in late life.The most effective means for reducing disease burden and control costs stays in delaying this progression, by extending "health-span", i.e. the period of human life, during which one is generally healthy and free from serious diseases. Accordingly, the effectiveness of stem/progenitor cell treatments, for delaying ageing process and age-related diseases, is an urgent necessity. We hope to see significant progresses in funding and support towards a comprehensive tool kit of rejuvenation therapies, currently not adequately supported. It is nonetheless encouraging to see the progresses reported in literature, as well as in papers in press in this journal.

This issue features a number of reviews and research's papers on the role of a particular type of progenitor cells, the endothelial progenitor cells (EPCS), in ageing, cells involved in the homeostasis and disease of endothelium, which it has become the main object of study in recent years. Precisely, the study of its dysfunction, "endothelium dysfunction", and the related cellular and molecular mechanisms has currently obtained the major interest in a large number of research groups. This is in accordance to well recognized evidence that endothelium dysfunction is the first pathological condition associated not only with all cardiovascular diseases (CVDs), but also with other age-related diseases, being the endothelial cells (ECs) components of the stroma of all tissues and organs. Accumulation of EC damages followed by death and impaired cardiovascular self repair, due principally to altered age-related function of tissuespecific resident and circulating stem or progenitor cells, such as EPCs, characterize the onset of endothelial dysfunction. Precisely, when an imbalance between dam-age/death and regeneration of the endothelium occurs, integrity and function of endothelium is threatened, and the origin and progression of CVDs, or other agerelated diseases, is favoured.

In this issue, a large range of current directions in research about this topic will be described and discussed. Thus, we think that the papers of this issue could be of interest to the readers of this journal:

Gambini and colleagues will describe the bone marrow ageing and its implications on EPC ageing and effects of senescent EPCs in cell therapy, summarizing potential anti-senescence strategies (Rurali et al., 2016).

Ruvolo and colleagues will summarize the recent literature in the field of vascular ageing, by discussing the major mechanisms involved in endothelial cell senescence, and underlining the key molecular aspects of ageing-associated vascular dysfunction in the attempt to highlight potential innovative therapeutic targets to delay the onset of age-related diseases (Regina et al., 2016).

Novo and co-authors will describe the age-related impairment of cardiovascular self-repair. In particular, they will illustrate ageing and age-related factors, which can affect cardiovascular repair by evocating the development of an imbalance between damage and self-repair, as risk factor for the onset and progression of CVDs. Particular relevance will be due in describing the role of ageing of cardiac stem and EPC cells (Madonna et al., 2016).

Provinciali and colleagues will examine the effect of both ageing and early stage of streptozotocin-induced diabetes on the number of bone marrow-derived $\mathrm{CD} 117^{+}$progenitor cells, and on their differentiation in vitro toward EPCs. They will demonstrate that some of the EPCs functional alterations induced by hyperglycaemia in young mice are observed in normal aged mice (Pierpaoli et al., 2016)

De Falco and coworkers will describe as EPC impairment associated with age contributes to doxorubicin-induced cardiotoxicity. In particular, they will evidence as Doxorubicin accelerates EPC ageing. They will demonstrate that Nox2 activity efficiently contributes to the mechanism of oxidative stress-induced increase in premature aging conferred by doxorubicin. Therefore, they will underline the relevance of Nox2 modulation in human EPCs as a useful tool to restore EPC physiological function and properties (De Falco et al., 2016).

Guggino and colleagues will summarize the literature data on the role of EPCs in the onset of specific age-related diseases, such as autoimmune disorders. Potential interventions will be suggested (Ferrante et al., 2016).

Altabas and colleagues will particularly discuss on therapeutic drug interventions (including lipid lowering drugs, antihypertensive agents, drug used in diabetes, hormones, prostaglandins and etc.) as potential measures for both improving number and functionality of EPCs, delaying the rate of ageing and consequently the onset of the most common age-related diseases, i.e. atherosclerosis (Altabas et al., 2016).

Candore and co-authors will emphasize Mediterranean nutraceutical foods as strategy for enhancing EPC number and functionality in ageing, and consequently improving vascular functionality during aging (Accardi et al., 2016).

Recchioni and coworkers underline the importance of physical activity in improving number and functionality of EPC cells. In particular, they will summarize the current knowledge on the contribution of EPCs to endothelial repair mechanisms in patients with chronic heart failure, focusing on the effects induced by exercise training, and hypothesizing that some of these effects can be mediated by epigenetic mechanisms, i.e. microRNA modulation (Recchioni et al., 2016).

\section{References}

Accardi, G., Aiello, A., Gambino, C.M., Virruso, C., Caruso, C., Candore, G., 2016. Mediterranean nutraceutical foods: strategy to improve vascular ageing. Mech. Ageing Dev. 159, 63-70, pii: S0047-6374(16)30011-2.

Altabas, V., Altabas, K., Kirigin, L., 2016. Endothelial progenitor cells (EPCs) in ageing and age-related diseases: how currently available treatment modalities affect EPC biology, atherosclerosis, and cardiovascular outcome. Mech. Ageing Dev. 159, 49-62, pii: S0047-6374(16)30014-8.

De Falco, E., Carnevale, R., Pagano, F., Chimenti, I., Fianchini, L., Bordin, A., Siciliano, C., Monticolo, R., Equitani, F., Carrizzo, A., Peruzzi, M., Vecchione, C., Rubattu, S. Sciarretta, S., Frati, G., 2016. Role of NOX2 in mediating doxorubicin-induced senescence in human endothelial progenitor cells. Mech. Ageing Dev. 159, 37-43, http://dx.doi.org/10.1016/j.mad.2016.05, pii: S0047-6374(16)30065-3.

Ferrante, A., Guggino, G., Di Liberto, D., Ciccia, F., Cipriani, P., Balistreri, C.R., Sireci, G., Giacomelli, R., Triolo, G., 2016. Endothelial progenitor cells: are they displaying a function in autoimmune disorders? Mech. Ageing Dev. 159, 44-48, pii: S0047-6374(16)30056-2.

Madonna, R., Novo, G., Balistreri, C.R., 2016. Cellular and molecular basis of the imbalance between vascular damage and repair in ageing and age-related diseases: as biomarkers and targets for new treatments. Mech. Ageing Dev. 159, 22-30, pii: S0047-6374(16)30026-4.

Pierpaoli, E., Moresi, R., Orlando, F., Malavolta, M., Provinciali, M., 2016. Effect of hyperglycemia on the number of CD117(+) progenitor cells and their differentiation toward endothelial progenitor cells in young and old ages. Mech. Ageing Dev. 159, 31-36, pii: S0047-6374(16)30012-4.

Recchioni, R., Marcheselli, F., Antonicelli, R., Lazzarini, R., Mensà, E., Testa, R., Procopio, A.D., Olivieri, F., 2016. Physical activity and progenitor cell-mediated endothelial repair in chronic heart failure: is there a role for epigenetics? Mech. Ageing Dev. 159, 71-80, pii: S0047-6374(16)30029 -X.

Regina, C., Panatta, E., Candi, E., Melino, G., Amelio, I., Balistreri, C.R. Annicchiarico-Petruzzelli, M., Di Daniele, N., Ruvolo, G., 2016. Vascular ageing and endothelial cell senescence: molecular mechanisms of physiology and diseases. Mech. Ageing Dev. 159, 14-21, pii: S0047-6374(16)30059-8.

Rurali, E., Bassetti, B., Perrucci, G.L., Zanobini, M., Malafronte, C., Achilli, F., Gambini, E., 2016. BM ageing: implication for cell therapy with EPCs. Mech. Ageing Dev. 159, 4-13 (pii: S0047-6374 30044-6). 


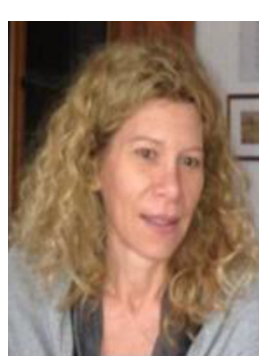

Fabiola Olivieri

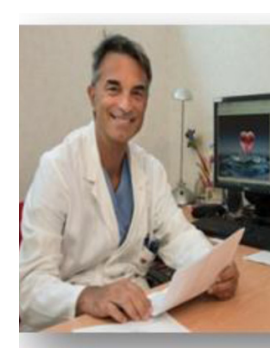

Giulio Pompilio

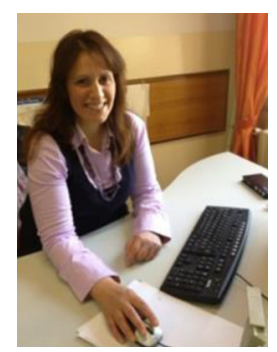

Guest Editor

Fabiola Olivieri

Department of Clinical and Molecular Sciences, DISCLIMO, Università Politecnica delle Marche,

Ancona, Italy

Guest Editor

Giulio Pompilio

Unit of Vascular Biology and Regenerative Medicine, Centro Cardiologico Monzino-IRCCS, Via Parea 4, 20138 Milan, Italy

Guest Manager Editor Carmela Rita Balistreri*

Department of Pathobiology and Medical Biotechnologies, University of Palermo, 90134

Palermo, Italy

*Corresponding author. E-mail address: carmelarita.balistreri@unipa.it (C.R. Balistreri)

Carmela Rita Balistreri

Available online 12 September 2016 